\title{
Vacuum Sealing Drainage in the Treatment of Migrated Polyacrylamide Hydrogel after Breast Augmentation: A Case Report
}

\author{
Jinfang Wu Xudong Zhang Qiming Zhao Dongsheng Mao Xin Lu
}

Department of Plastic and Reconstructive Surgery, No. 117 PLA Hospital, Hangzhou, China

\section{Established Facts}

- Polyacrylamide hydrogel (PAAG) injection for breast augmentation can result in a variety of complications, including implant migration and secondary infections.

- Treatment of such complications can be extremely difficult.

\section{Novel Insights}

- Vacuum sealing drainage can be used to repair a variety of chronically non-healing wounds and shorten the course of disease.

\section{Keywords}

Vacuum sealing drainage . Polyacrylamide hydrogel . Breast augmentation - Complications

\section{Summary}

Background: Polyacrylamide hydrogel (PAAG) injection for breast augmentation can result in a variety of complications, including implant migration. The migrated PAAG can become colonized by pathogens, resulting in secondary infections which can be extremely difficult to treat. Case Report: We report the case of a 43-year-old female patient who underwent PAAG injection for breast augmentation. Implant migration was diagnosed 8 years after the initial injection. Conclusion: Here, we report the first use of vacuum sealing drainage as a convenient and effective alternative to manage the complications of extensive PAAG migration after breast augmentation.

\section{Introduction}

Polyacrylamide hydrogel (PAAG), a polymer of the acrylamide monomer, was once thought to be a safe and biocompatible soft tissue filler. It has been widely used in cosmetic procedures, such as breast augmentation and facial contouring, for more than 10 years. However, a number of studies have reported complications of PAAG injection for breast augmentation, including induration of breast tissue, lump formation, hematoma, inflammation and infection, persistent breast pain, glandular atrophy, and gel migration [1]. Among these complications, indurations and the formation of lumps are the most common. In particular, extensive migration of implant material and consequent secondary infections are complex and often difficult complications to manage. Previous studies have reported several approaches to treat the complications of PAAG injection, including negative pressure suction, surgical removal of hydrogel-infiltrated tissues, open drainage, and intermittent lavage [2]. Here, we introduced the novel method of vacuum sealing drainage (VSD), and report the case of a patient whose

\section{KARGER}

Fax +497614520714

Information@Karger.com

www.karger.com

\section{(C) 2014 S. Karger GmbH, Freiburg}

$1661-3791 / 14 / 0094-0273 \$ 39.50 / 0$

Accessible online at:

www.karger.com/brc
Xudong Zhang, MD

Department of Plastic and Reconstructive Surgery No. 117 PLA Hospital

No. 14, Lin Ying Rd., Hangzhou 310013, PR China zxdyqky@yahoo.com.cn 
Fig. 1. Magnetic resonance images showing polyacrylamide hydrogel (PAAG) A as a low signal on T1weighted images and B as a high signal on T2-weighted images

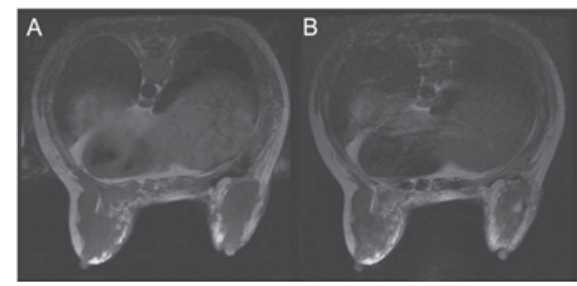

in both breasts. The images show a structural disorder of both breasts with nodular or cord-like lesions in the glandular and ductal structures. A copious amount of gel-like material was still observed in both breasts.
Fig. 2. A, B

Preoperative view (established under ultrasound guidance) of polyacrylamide hydrogel (PAAG)induced lumps and cystic cavities of varying sizes in the mammary and subcutaneous tissues, pectoral fascia space of the chest, and abdominal regions.
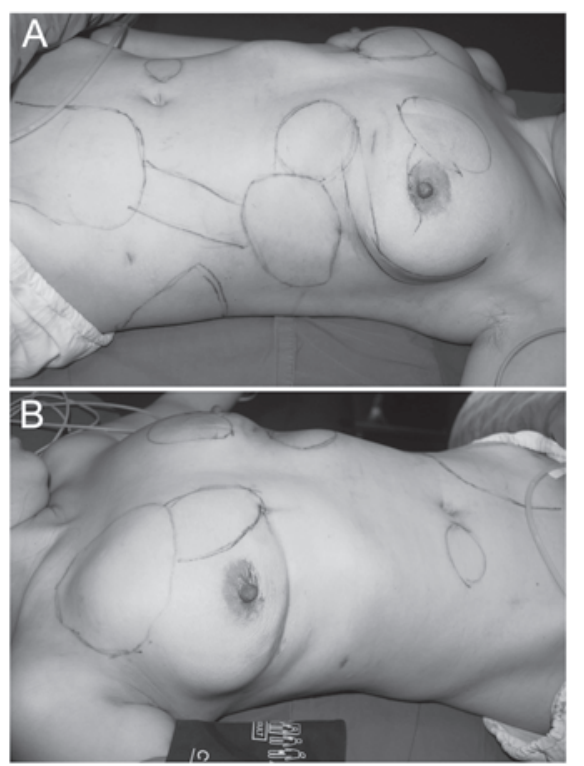
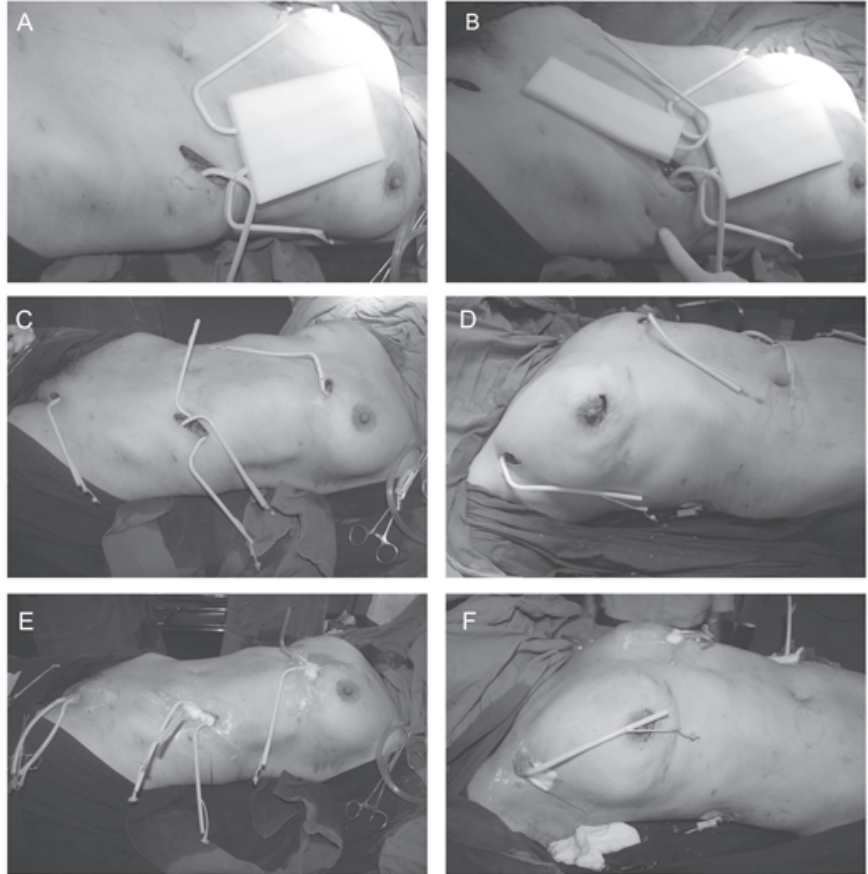

Fig. 3. Vacuum sealed drainage (VSD) procedure for the treatment of migrated polyacrylamide hydrogel (PAAG). A, B 2 size specifications of VSD dressings $(15 \times 10 \mathrm{~cm}$ and $15 \times 5 \mathrm{~cm})$ were used based on the size of the wound cavity. C, D VSD dressings were used to fill the wound cavities, with one end of the drainage tube embedded in the dressings and the other end left external to the dressing. E, F Wounds were then completely sealed with biological semipermeable membranes. The drainage tube was connected to a negative pressure system.
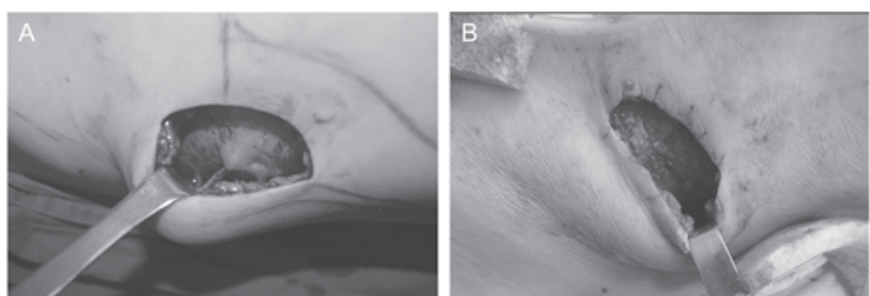

Fig. 4. A The wound cavity under the left side of the abdominal wall was separated by fibrous cords and vascular perforating branches through which tunnels had formed. The internal walls were covered with exudates and necrotic tissue, creating a smooth membrane-like material. B After 1 week of VDS treatment, the membrane-like material on the inner walls had disappeared, and granulation tissue had started to grow within the wound; however, the fluid draining from the wounds remained opaque and turbid. The VSD dressings were changed and remained in place for a further week before being removed. breasts, the left side of the abdomen, waist and vulva, and were occasionally accompanied by systemic reactions such as fever. Laboratory tests showed a white blood count of $16.19 \times 10^{9} / 1$, a neutrophil count of $13.98 \times$ $10^{9} / 1$, and an erythrocyte sedimentation rate of $86.0 \mathrm{~mm} / \mathrm{h}$, which indicated an existing infection. Magnetic resonance imaging examination of both breasts indicated disordered glandular and ductal structures (fig. 1).

Guided by ultrasound imaging, the sites of the migrated implants were marked on the chest and abdomen (fig. 2). Small incisions $(3-5 \mathrm{~cm})$ were made on the right areola, the skin folds under the left breast, and at the waist on the left anterior axillary line. Cysts of various sizes were observed within the subcutaneous, glandular, and muscular layers. During surgery, membrane-like materials and fibrous tissue within the inner cyst walls were completely removed and sent to a pathology laboratory for rapid analysis. The wound cavities were filled with VSD dressings (polyvi- nyl alcohol sponge dressings with the end of the drainage tube embedded in the sponge), then sealed with a biological semipermeable membrane. The other end of the drainage tube was directly connected to a negative pressure suction system maintained at $500-600 \mathrm{mmHg}$, thus creating a negative pressure environment (fig. 3). Each wound cavity was numerically labeled, and changes in the quality and quantity of drainage were observed and recorded. The pathological results showed the presence of chronic inflammation.

A week later, the drainage fluid from the breast subcutaneous space became clear. Granulation tissue was growing well, and the wound infec- 
Fig. 5. One week after the removal of all drainage tubes, clinical symptoms including redness and swelling had vanished, and the wound cavities in both breasts, chest, abdomen, inguinal area, and vulva had completely healed.

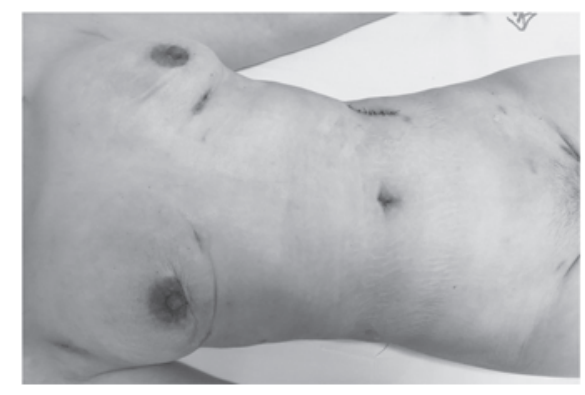

tions were controlled. The VSD dressings were removed, but the drain age catheter was left in situ in each wound for a further 3 days. However, the drainage fluid from the wounds on the abdominal wall remained opaque and turbid, while granulation tissue had started to grow within the wound (fig. 4). The VSD dressings were changed, and remained in place for a further week before being removed. 1 week after the removal of all drainage tubes, clinical symptoms including redness and swelling had vanished, and all wounds were completely healed (fig. 5). The patient was followed-up for 6 months without any recurrence of her previous symptoms, and she was satisfied with the outcome of the VSD treatment.

\section{Discussion}

The VSD method shares common principles with negative pressure wound therapy and vacuum-assisted closure techniques [3]. To date, VSD has not only been used clinically for the treatment of superficial wounds, but also has therapeutic effects in deep wound drainage. VSD has shown certain advantages compared to other surgical drainage methods, as the
VSD dressings entirely fill the wound cavity, which helps to eliminate bacteria and residual toxic substances [4]. In the present study, we found that VSD dressings would gradually decrease the volume of exudates and alleviate infections and inflammatory reactions, thus effectively controlling sepsis. Exudative material such as fibronectin were quickly and effectively removed, avoiding the formation of fibers in the lacunar wall, which prevents wound healing. Moreover, the continuous negative pressure suction creates a negative-pressure environment within the wound cavity, which makes the inner walls of the wound become tightly adherent to each other. Thus, after the removal of the VSD dressings, we found the wound cavities to be completely closed and healing very rapidly. In addition, continuous negative pressure suction may increase local blood flow, and promote angiogenesis and the growth of granulation tissue around the wound, which facilitates wound healing $[5,6]$. The application of VSD for the treatment of migrated PAAG after breast augmentation has not been reported previously.

In conclusion, VSD is a convenient and effective treatment for dealing with the complications of extensive PAAG migration after breast augmentation, especially when accompanied by chronic infection and refractory to conventional treatments.

\section{Disclosure Statement}

The authors declare that they have no conflict of interest.

\section{References}

1 Do ER, Shim JS: Long-term complications from breast augmentation by injected polyacrylamide hydrogel. Arch Plast Surg 2012;39:267-269.

2 Yu L, Wang J, Zhang B, Zheng DN, Zhu C: Treatment of breast injection with polyacrylamide hydrogel with infiltrated fascia capsule removal: report on 104 cases. Aesthetic Plast Surg 2012;36 $1120-1127$.
Moues CM, Heule F, Hovius SE: A review of topical negative pressure therapy in wound healing: sufficient evidence? Am J Surg 2011;201:544-556.

4 Weed T, Ratliff C, Drake DB: Quantifying bacterial bioburden during negative pressure wound therapy: does the wound vac enhance bacterial clearance? Ann Plast Surg 2004;52:276279; discussion 279-280.
5 Chen SZ, Li J, Li XY, Xu LS: Effects of vacuumassisted closure on wound microcirculation: an experimental study. Asian J Surg 2005;28:211-217.

6 Labler L, Rancan M, Mica L, Harter L, MihicProbst D, Keel M: Vacuum-assisted closure therapy increases local interleukin-8 and vascular endothelial growth factor levels in traumatic wounds. J Trauma 2009;66:749-757. 